\title{
Expanding the usefulness of hemodynamic waveform analysis in the critically III
}

\author{
Michael R. Pinsky ${ }^{1}$
}

Received: 15 December 2018 / Accepted: 16 December 2018 / Published online: 2 January 2019

(c) Springer Nature B.V. 2019

Right ventricular (RV) function is usually not important clinically in defining cardiovascular status until it is. The role RV function plays in maintaining normal cardiovascular homeostasis defines that as long as it maintains a low right atrial pressure (Pra) in response to circulatory stresses like exercise, acute respiratory failure, or sepsis, its function can be ignored [1]. Under normal conditions pulmonary arterial systolic pressure remain low, usually below $16 \mathrm{mmHg}$, such that the ejecting RV can transfer its blood easily into the pulmonary arterial bed. Ventilation, by altering both intrathoracic pressure (ITP) and lung volume, affects this process in multiple ways that include varying right atrial pressure independent of RV filling pressures and pulmonary vascular resistance. Spontaneous inspiration by decreasing ITP artifactually decreases Pra while simultaneously increasing the rate of RV filling [2]. This is good because if RV filling pressure does rise with increased filling, Pra does not, thus fulfilling the requirements of an adaptive RV in sustaining a maximal venous return. Positive pressure breathing, regrettably does the exact opposite, it increases ITP artifactually increasing Pra and impeding venous return. Thus, increased Pra values are commonly seen in patients on mechanical ventilation even if they have normal RV function. Still, many disease states commonly seen in the acute care setting are associated with increased pulmonary vascular resistance. Operationally, this is compensated for reflexively by fluid retention and fluid resuscitation by the clinician. Thus, in the setting of acute illness Pra is commonly elevated but its relationship to RV function is unknown. Presently, echocardiographic analysis of right ventricular function and ventricular interdependence are used to assess RV function

Michael R. Pinsky

pinsky@pitt.edu

1 Department of Critical Care Medicine, University of Pittsburgh School of Medicine, 1215.4 Kaufmann Medical Building, 3471 Fifth Avenue, Pittsburgh, PA 15213, USA at the bedside, but these methods are user dependent, not universally available or available all the time, cannot be easily used to continually followed RV function as time or treatments occur, and define only a specific state not the response to stress or cardiovascular reserve, which are what are really needed for the bedside assessment of RV function to be clinically useful.

Presently dynamic estimates of volume responsiveness, including pulse pressure variation (PPV), degrade with marked RV failure [3]. Indeed, PPV can be $>10 \%$ in severe heart failure but $180^{\circ}$ out-of-phase because inspirationinduced decreased RV overload allowing LV end-diastolic volume to increase. Still, few clinicians view the phase relation of PPV to the ventilatory cycle, making this distinction less useful. Alternatively one could give a small volume challenge. If Pra increases $>2 \mathrm{mmHg}$ to a $250 \mathrm{~mL}$ fluid bolus, RV dysfunction is probable [4]. Still this would require repeated fluid challenges and could lead to volume overload. If one could use a simple monitoring approach to assess RV function without the need for ventilator phase analysis or a fluid challenge, such a test would be useful in the monitoring and potential management of critically ill patients.

Within this context the study by Vistisen et al. in this issue becomes relevant [5]. The authors reasoned that since $\mathrm{RV}$ dysfunction should be associated with an inability to increase RV stroke volume in response to increased filling, if its impact on subsequent left ventricular (LV) filling could be assessed, then the selective increases in LV output associated with increasing RV filling would identify volume responsive patients, whereas a lack of increase in LV output would identify combined RV and LV dysfunction. To pilot that hypothesis they chose to examine change in systolic arterial pressure in the subsequent $2 \mathrm{nd}$ and $3 \mathrm{rd}$ heart beats following a premature ventricular contraction (PVC) from the large retrospective MIMIC-III database for patients with both arterial pressure waveforms, existence of PVCs during those recordings and an associated within 
$12 \mathrm{~h}$ echocardiographic examination confirming either RV dysfunction or normal RV function (controls). Of the 2086 subjects with echocardiographic reports they identified 93 and 66 control and RV dysfunction PVC event, respectively, from which they took a subgroup of 24 controls and $34 \mathrm{RV}$ dysfunction patients. To quantify the dynamic changes in LV output that measured systolic arterial pressure for the 10 sinus beats preceding the PVC as baseline and the 2 nd and 3 rd post-PVC beats as the output $\left(\Delta \mathrm{SBP}_{\text {beat 2-3 }}\right)$. The $\triangle \mathrm{SBP}_{\text {beat 2-3 }}$ for controls was $-3.6(1.9) \%$ and for RV dysfunction patients $-1.7(1.9) \%(\mathrm{p}<0.001)$ with the best performance of their parameter above the "grey zone" at $-1.5 \%$ which gave a high specificity (91\%) but a 50\% sensitivity. They conclude that this proof-of-concept study documented that PVC arterial blood pressure characteristics are influenced by RV dysfunction.

This study has two positive aspects to its findings and their implications. First of all, since this process can be automated to assess any patient being monitored with an arterial waveform output, RV dysfunction and its change can potentially be assessed both in individual patients and over time. Second, the use of arrhythmias instead of positive pressure breaths or passive leg raising maneuvers to alter RV filling means that this analysis can be done on any such monitored patient or, as in this case, any large retrospective database.

Regrettably, this study also has several major limitations that will need to be addressed if such "passive" analysis is to be used going forward. First of all, PVCs are rare and cannot be counted upon to occur uniformity across monitored patient populations. Still, if present, they can be leveraged. Second, the authors used systolic pressure to calculate their DeltaSBP $_{\text {beat 2-3 }}$ parameter. As shown in their Figs. 2 and 3 , systolic arterial pressure is greatly influenced by changes in ITP. Considering as how RV dysfunction diagnosis required a $-1.5 \%$ change, such influences are not trivial. Although the baseline 10-sinus beat reference values can minimize this effect on baseline, they cannot remove ventilatory artifact from a 2-beat post PVC analysis. Potentially, this limitation could be minimized by their using arterial pulse pressure instead, because it is minimally affected by ITP at these low levels of change. Third, the etiology of volume responders and non-responders was not addressed in this study. For example, we do not know what the baseline PPV of the two groups were. LV dysfunction alone should also make $\Delta \mathrm{SBP}_{\text {beat 2-3 }}$ less than normals. Finally, RV dysfunction is not one diagnosis or etiology and acute illness can affect RV function in a variety of ways from changes in pulmonary vascular resistance, impedance, and compliance to ischemic dysfunction. Thus, finding of reduced $\Delta \mathrm{SBP}_{\text {beat 2-3 }}$ may become an alerting signal to explore cardiovascular dysfunction further or merely underlying known existing dysfunction. The proof will be in the use of these dynamic parameters for both classification of disease and decisions about a more personalized treatment approach. It will be interesting to see if this approach or others using similar paradigms gain purchase in the monitoring and classification of cardiovascular dysfunction in the critically ill.

Funding Funding was provided by NIH GM117622.

\section{Compliance with ethical standards}

Conflict of interest The author declare that they have no competing of interest.

\section{References}

1. Guyton AC, Lindsey AW, Abernathy B, Richardson T. Venous return at various right atrial pressures and the normal venous return curve. Am J Physiol. 1957;189:609-15.

2. Mahmood SS, Pinsky MR. Heart-lung interactions during mechanical ventilation: the basics. Ann Transl Med. 2018;6:349.

3. Daudel F, Tüller D, Krähenbühl S, Jakob SM, Takala J. Pulse pressure variation and volume responsiveness during acutely increased pulmonary artery pressure: an experimental study. Crit Care. 2010;14:R122.

4. Pinsky MR, Kellum JA, Bellomo R. The CVP is a stop rule, not a target of resuscitation. Crit Care Resusc. 2014;16:245-6.

5. Vistisen ST, Moody B, Celi LA, Chen C. Post-extrasystolic characteristics in the arterial blood pressure waveform are associated with righ ventricular dysfunction in intensive care patients. J Clin Monit Comput. 2018;1-7. 